\title{
REVIEW ARTICLE ON GENE THERAPY
}

\author{
PATIL P.M. ${ }^{1}$, CHAUDHARI P.D. ${ }^{1}$, MEGHA SAHU1 AND DURAGKAR N.J. ${ }^{2}$ \\ ${ }^{1}$ Modern College of Pharmacy, Sector 21, Yamunanagar, Nigdi, Pune-44, MS, India. \\ 2Sharad Pawar College of Pharmacy, Nagpur, MS, India. \\ ${ }^{*}$ Corresponding Author: Email- pallavipatil_2007@yahoo.com¹, psadanshio@yahoo.co.In²
}

Received: May 26, 2011; Accepted: March 15, 2012

\begin{abstract}
Gene therapy can be broadly defined as the transfer of genetic material to cure a disease or at least to improve the clinical status of a patient. One of the basic concepts of gene therapy is to transform viruses into genetic shuttles, which will deliver the gene of interest into the target cells. Based on the nature of the viral genome, these gene therapy vectors can be divided into RNA and DNA viral vectors. The majority of RNA virus-based vectors have been derived from simple retroviruses like murine leukemia virus. A major shortcoming of these vectors is that they are not able to transducer nondividing cells. This problem may be overcome by the use of novel retroviral vectors derived from lentiviruses, such as human immunodeficiency virus (HIV). The most commonly used DNA virus vectors are based on adenoviruses and adeno-associated viruses. An example of gene-knockout mediated gene therapy is the knockout of the human CCR5 gene in T-cells in order to control HIV infection[1] Although the available vector systems are able to deliver genes in vivo into cells, the ideal delivery vehicle has not been found. Thus, the present viral vectors should be used only with great caution in human beings and further progress in vector development is necessary.
\end{abstract}

Key words- Lentiviruses, transducer, RNA vector, adeno-associated viruses.

Citation: Patil P.M., et al. (2012) Review Article on Gene Therapy. International Journal of Genetics, ISSN: 0975-2862 \& E-ISSN: 09759158, Volume 4, Issue 1, pp.-74-79.

Copyright: Copyright@2012 Patil P.M., et al. This is an open-access article distributed under the terms of the Creative Commons Attribution License, which permits unrestricted use, distribution, and reproduction in any medium, provided the original author and source are credited.

\section{Introduction}

Gene therapy is a technique for correcting defective genes responsible for disease development. Researchers may use one of several approaches for correcting faulty genes:

- A normal gene may be inserted into a nonspecific location within the genome to replace a nonfunctional gene. This approach is most common.

- An abnormal gene could be swapped for a normal gene through homologous recombination.

- The abnormal gene could be repaired through selective reverse mutation, which returns the gene to its normal function.

- Gene therapy is the insertion, alteration, or removal of genes within an individual's cells and biological tissues to treat diseases. It is a technique for correcting defective genes that are responsible for disease development.

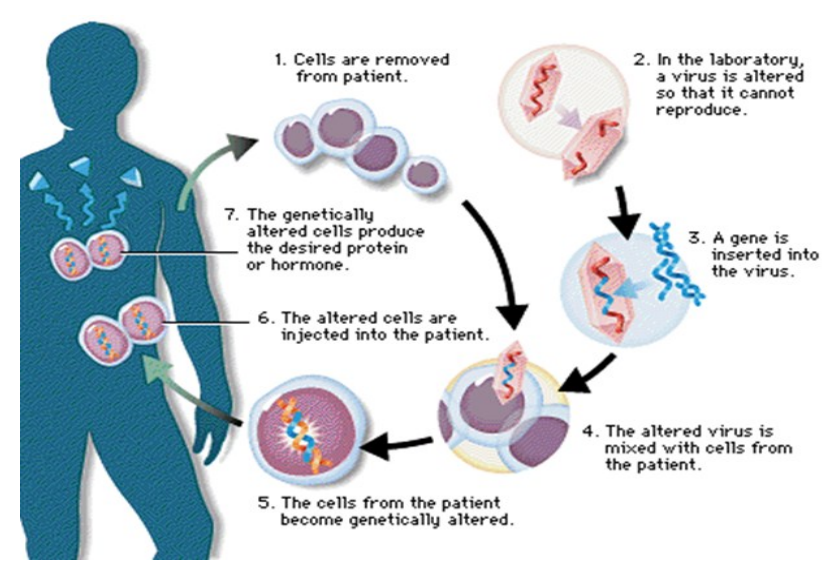

Fig. 1- Flow chart shows gene therapy 


\section{Gene therapy history and future}

The girl was treated on 14th September, 1990, at the National Institutes of Health's Clinical Center, Bathesda, Maryland. Dr. W. French Anderson and his colleagues at the health center, carried out the proceedings. White blood cells were extracted from the body. After the implantation of genes that produce ADA, the cells were transferred back to the girl's body. Considerable improvement in the immune system of the girl was noticed. Meanwhile, the trials of gene therapy continued on various diseases. The patients with skin cancer, melanoma were treated by means of gene therapy.

\section{Principle of gene therapy}

- An abnormal gene could be swapped for a normal gene through homologous recombination.

- The abnormal gene could be repaired through selective reverse mutation, which returns the gene to its normal function.

- The regulation (the degree to which a gene is turned on or off) of a particular gene could be altered.

\section{Approaches of gene therapy}

1. Gene modification

- Replacement therapy

- Corrective Gene therapy

2. Gene transfer

- Physical

- Chemical

- Biological

3. Gene transfer in specific cell line

- Somatic gene therapy

- Germ line gene therapy

4. Eugenic approach (gene insertion)

Other forms of genetic engineering include gene targeting and knocking out specific genes via engineered nucleases such as zinc finger nucleases, engineered I-Crel homing endonucleases, or nucleases generated from TAL effectors. This approach is currently being used in several human clinical trials.[2]

\section{Vectors in gene therapy}

Some of the different types of viruses used as gene therapy vectors:

Retroviruses- A class of viruses that can create double-stranded DNA copies of their RNA genomes. These copies of its genome can be integrated into the chromosomes of host cells. Human immunodeficiency virus (HIV) is a retrovirus.

eg:- One of the problems of gene therapy using retroviruses is that the integrase enzyme can insert the genetic material of the virus into any arbitrary position in the genome of the host; it randomly inserts the genetic material into a chromosome. If genetic material happens to be inserted in the middle of one of the original genes of the host cell, this gene will be disrupted (insertional mutagenesis). If the gene happens to be one regulating cell division, uncontrolled cell division (i.e., cancer) can occur. This problem has recently begun to be addressed by utilizing zinc finger nucleases[3] or by including certain sequences such as the beta-globin locus control region to direct the site of integration to specific chromosomal sites.
- Adenoviruses- A class of viruses with double-stranded DNA genomes that cause respiratory, intestinal, and eye infections in humans. The virus that causes the common cold is an adenovirus.

- Adeno -associated viruses- A class of small, single-stranded DNA viruses that can insert their genetic material at a specific site on chromosome 19.

- Cis and trans-acting elements- Replication-defective vectors always contain a "transfer construct". The transfer construct carries the gene to be transduced or "transgene". The transfer construct also carries the sequences which are necessary for the general functioning of the viral genome: packaging sequence, repeats for replication and, when needed, priming of reverse transcription. These are denominated cis-acting elements, because they need to be on the same piece of DNA as the viral genome and the gene of interest.[4]

- Herpes simplex viruses- A class of double-stranded DNA viruses that infect a particular cell type, neurons. Herpes simplex virus type 1 is a common human pathogen that causes cold sores.[5]

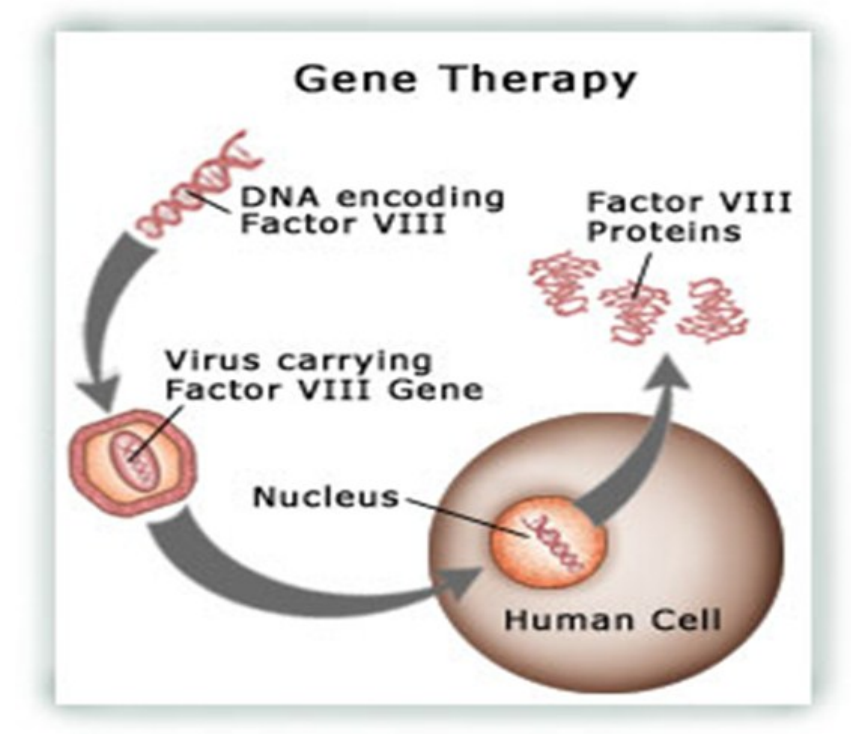

Fig. 2- Viral method of gene therapy

\section{Non-viral methods}

- Non-viral methods present certain advantages over viral methods,

- with simple large scale production and low host immunogenicity being just two. Previously, low levels of transfection and expression of the gene held non-viral methods at a disadvantage; however, recent advances in vector technology have yielded molecules and techniques with transfection efficiencies similar to those of viruses.

- Ormasil (organically modifided silica or silicate) used as nonviral method.

\section{Injection of Naked DNA}

This is the simplest method of non-viral transfection. Clinical trials carried out of intramuscular injection of a naked DNA plasmid have occurred with some success; however, the expression has been very low in comparison to other methods of transfection. 
Liposome for Drug Delivery

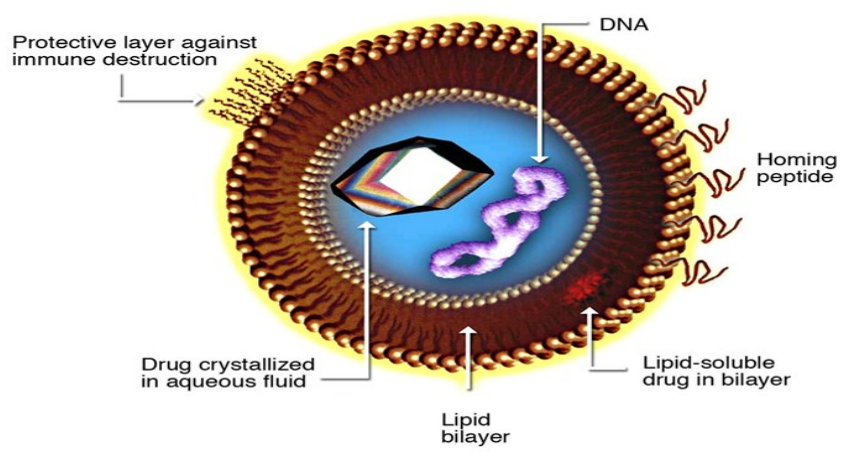

Fig. 3- Liposome for drug delivery[6]

\section{Physical Methods to Enhance Delivery}

- Electroporation- Electroporation is a method that uses short pulses of high voltage to carry DNA across the cell membrane. This shock is thought to cause temporary formation of pores in the cell membrane, allowing DNA molecules to pass through. Electroporation is generally efficient and works across a broad range of cell types. However, a high rate of cell death following electroporation has limited its use, including clinical applications.

- Gene Gun- The use of particle bombardment, or the gene gun, is another physical method of DNA transfection. In this technique, DNA is coated with gold particles and loaded into a device which generates a force to achieve penetration of DNA/ gold into the cells.eg:- If the DNA is integrated in the wrong place in the genome, for example in a tumor suppressor gene, it could induce a tumor. This has occurred in clinical trials for $X$ -linked severe combined immunodeficiency (X-SCID) patients, in which hematopoietic stem cells were transduced with a corrective transgene using a retrovirus, and this led to the development of T cell leukemia in 3 of 20 patients.[7]

- Sonoporation- Sonoporation uses ultrasonic frequencies to deliver DNA into cells. The process of acoustic cavitation is thought to disrupt the cell membrane and allow DNA to move into cells.

- Magnetofection- In a method termed magnetofection, DNA is complexed to a magnetic particles, and a magnet is placed underneath the tissue culture dish to bring DNA complexes into contact with a cell monolayer.

\section{Chemical Methods to Enhance Delivery}

- Oligonucleotides- The use of synthetic oligonucleotides in gene therapy is to inactivate the genes involved in the disease process. There are several methods by which this is achieved. One strategy uses antisense specific to the target gene to disrupt the transcription of the faulty gene. Another uses small molecules of RNA called siRNA to signal the cell to cleave specific unique sequences in the mRNA transcript of the faulty gene, disrupting translation of the faulty mRNA, and therefore expression of the gene.

- Lipoplexes and polyplexes- To improve the delivery of the new DNA into the cell, the DNA must be protected from damage and (positively charged). Initially, anionic and neutral li- pids were used for the construction of lipoplexes for synthetic vectors.

- Dendrimers- A dendrimer is a highly branched macromolecule with a spherical shape. The surface of the particle may be functionalized in many ways and many of the properties of the resulting construct are determined by its surface. In particular it is possible to construct a cationic dendrimer, i.e. one with a positive surface charge. When in the presence of genetic material such as DNA or RNA, charge complimentarity leads to a temporary association of the nucleic acid with the cationic dendrimer. On reaching its destination the dendrimer-nucleic acid complex is then taken into the cell via endocytosis.

- Hybrid methods- Due to every method of gene transfer having shortcomings, there have been some hybrid methods developed that combine two or more techniques. Virosomes[8 ] are one example; they combine liposomes with an inactivated HIV or influenza virus. This has been shown to have more efficient gene transfer in respiratory epithelial cells than either viral or liposomal methods[9] alone. Other methods involve mixing other viral vectors with cationic lipids or hybridising viruses.

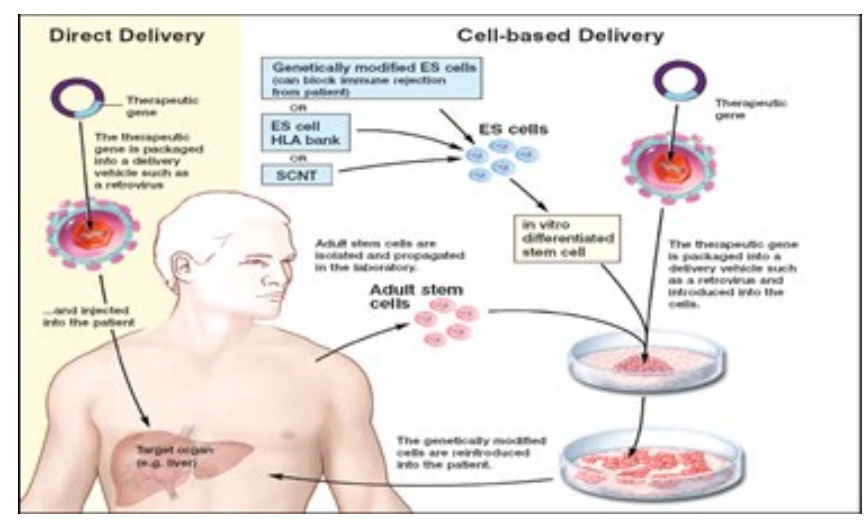

Fig. 4- Delivery of gene by direct and cell based[10] methods

\section{Advantages and disadvantages of gene therapy Advantages of gene therapy}

- In case of 'silence' a gene. In the case of someone with HIV, which had not yet developed into AIDS, scientists could save them the pain and suffering of the disease by using gene therapy to 'silence' the disease before its onset.

- Gene therapy has the potential to eliminate and prevent hereditary diseases such as cystic fibrosis and is a possible cure for heart disease, AIDS and cancer.

- These sceptics would almost certainly choose gene therapy, especially if it was the last hope for them or one of their loved ones - as is the case for many gene therapy patients.[11]

Disadvantages of Gene Therapy

- Short-lived nature of gene therapy.

- Immune response - Genes injected with a virus may trigger an immune response against the virus. Problems with viral vectors (once inside the patient, the viral vector could recover its ability to cause disease).

- Multigene disorders - The genetic material might not get into the right cell, or the right place in the cell's DNA.[12] 


\section{Ethical issues surrounding gene therapy are}

- Who decides which traits are normal and which constitute a disability or disorder?

- Will the high costs of gene therapy make it available only to the wealthy?

- Could the widespread use of gene therapy make society less accepting of people who are different?

- Should people be allowed to use gene therapy to enhance basic human traits such as height, intelligence, or athletic ability?

\section{Application of gene therapy \\ 1. In case of Parkinson's diseases}

Gene therapy has been proven to work for Parkinson's disease, The Independent has reported.A number of other newspapers also express the hope offered by the new procedure, which is intended to boost levels of a brain chemical called GABA, which is lacking in people with Parkinson's. In a small trial of the technique, 45 participants with severe disease had their brains implanted with tubes that led to areas of the brain that deal with movement. Half were injected with a virus carrying a gene that would increase GABA production. The other half were given a harmless saline solution. After six months, those treated with gene therapy showed a $23 \%$ improvement in movement, twice that seen among those given sham surgery.

This was a randomised controlled trial designed to investigate whether some symptoms of advanced Parkinson's disease could be improved with gene therapy, a relatively new experimental technique which can theoretically be used to introduce new genes into the body. In this case, gene therapy was used to transfer a gene for producing a chemical called glutamic acid decarboxylase (GAD) into the basal ganglia, a collection of brain areas that control movement. The GAD gene being introduced is involved in increasing levels of a signalling chemical called GABA. Levels of GABA are decreased in some parts of the basal ganglia in people with Parkinson's disease.

The trial was conducted as a 'proof of concept', which tested gene therapy against sham surgery. The patients allocated sham treatment received the same surgical implant as the gene therapy patients but no gene therapy. The trial was double-blind, meaning that neither the patients nor the researchers knew if the gene therapy or a sham treatment had been given. Further to this, the researchers were careful to take steps that would

Gene therapy provides new hope for Parkinson's patients-

Promising new research may provide new hope for patients with Parkinson's disease, doctors at Stanford University started treating patients with a new gene therapy treatment.

One woman says it stopped her shaking and enabled her to resume her active life. Parkinson's patient Sharon jokela in San Francisco is doing something she couldn't a year and a half ago...walk without shaking, she recalled, "I was shaking so much that I couldn't lift a fork or a wine glass. "Her tremors were so bad the graphic designer couldn't work and could barely drive. The active hiker found herself homebound most days, "It was horrible. I mean, I did think, 'I do not want to live like this. I would rather die."Medications offered little relief and lots of side effects, so Sharon volunteered for a clinical trial at Stanford medical center looking at the effectiveness of a new gene therapy treatment.

\section{In case of Alzheimer's disease}

Scientists have successfully switched off a gene thought to cause Alzheimer's by using a new way to deliver drugs directly to the brain," reported the Daily Mirror. The newspaper said that researchers have used "tiny particles called exosomes, which are released by cells, to administer drugs into the brains of mice". The laboratory study behind these headlines was carried out in mice. The findings are significant, demonstrating that exosomes could be used to carry gene therapy to particular genes in the brain. One of these genes is BACE1, which produces a protein associated with Alzheimer's disease.

The study paves the way for future research, and the finding will be of great interest to the scientific community. Exosomes appear to be able to deliver specific 'cargoes' to brain cells so the technology has a number of potential applications. However, this is early research and the technology has not been tested in human cells. There is also a range of technical and ethical issues associated with gene therapy in humans. Many neurological diseases involve degeneration and cell loss. Neurotrophic factors are proteins which promote cell growth in development and have been found to be neuroprotective in some neurological diseases, both in the central and peripheral nervous system, making them ideal candidates for gene therapy.

In 1988, NGF was the first therapeutic molecule to be administered using ex vivo gene therapy in an animal model of neurological disease. Since then, the field has been growing and, in the case of NGF, clinical trials are currently taking place involving ex vivo gene therapy in Alzheimer's disease.

GDNF in the brain:-Another neurotrophic factor which has generated much interest is GDNF. In the CNS, GDNF has been identified to be neuroprotective for a subset of dopaminergic neurones in the midbrain. This population of neurones degenerates in patients with Parkinson's disease, making GDNF a target for treatment possibilities. Several studies involving GDNF administration into the brain have successfully abolished behavioural signs of Parkinson's disease in animal models and shown prevention of degeneration of nigrostriatal dopaminergic pathways. The authors of this study propose that gene therapy could be even more successful in efficacy as their method of administration resulted in a relatively limited diffusion of GDNF in the putamen, possibly leaving more remote parts of this brain structure for continued degeneration.

Another lentiviral vector system was developed based on the EIAV (equine infectious anaemia virus. It has been efficiently used to transfect brains and spinal cords, the majority of transduced cells being of neuronal morphology and VSVG-EIAV mediated GDNF delivery has also been shown to be successful in animal models of Parkinson's disease..

Research into treatment of pain states, has concentrated mostly on the use of HSV vectors which can be applied in the periphery and retrogradely transported up to the dorsal root ganglia. A study using HSV mediated GDNF administration in the periphery was reported to be successful in alleviating pain and some neurochemical changes. Although this delivery method has great advantages in that it is minimally invasive - the virus can transfect neural cells even just through a scratch in the skin the downsides of using the HSV system are: 
1) Virus associated toxicity and immunoreactivity though with new methods of virus development, the toxicity and immune response inducing parts of the virus can be removed.

2) Replication of the virus in the transfected cells the relative short latency of expression.

Virally mediated spinal administration of several molecules has so far been successfully used in some models of pain. Eaton et al report neuroprotective effects of AAV-BDNF administration. GDNF has not yet been administered intraspinally in models of peripheral neuropathy. To date, only several studies looked at intraspinal injection of GDNF expressing viral vectors (Lv and AAV) in models of ALS and ventral root avulsion.

\section{In case of cyctic fibrosis}

In therapy, treatment targets the cause of cystic fibrosis rather than just treating the symptoms. Although the first gene therapy experiments have involved lung cells, scientists hope that these technologies will be adapted to treat other organs affected by cystic fibrosis.

- Cystic Fibrosis

- Causes of Cystic Fibrosis

- Cystic Fibrosis Gene

- Early Symptoms of Cystic Fibrosis

- Cystic Fibrosis Symptoms

- Cystic Fibrosis Diagnosis

- Cystic Fibrosis Sweat Test

- Prenatal Testing for Cystic Fibrosis

- Cystic Fibrosis Genetic Testing

- Treatment for Cystic Fibrosis

- Living with Cystic Fibrosis

- Cystic Fibrosis and Who It Affects

History of cystic fibrosis gene therapy:- Gene therapy for cystic fibrosis began in 1990, when scientists successfully corrected faulty cystic fibrosis transmembrane conductance regulator (CFTR) genes. They did this by adding normal copies of the gene to laboratory cell cultures. In 1993, the first experimental CF gene therapy treatment was given to a patient with cystic fibrosis. Researchers modified a common cold virus to act as a delivery vehicle by carrying normal genes to the CFTR cells in the airways of the lung.Subsequent studies have tested other methods of gene delivery, such as: fat capsules, synthetic vectors, nose drops, or drizzling cells down a flexible tube to CFTR cells lining the airways of lungs. Researchers are now testing aerosol delivery using nebulizers

Challenges of This Type of Therapy

Finding the best delivery system for transporting normal CFTR genes is only one challenge that scientists must solve to develop an effective treatment for cystic fibrosis. Scientists must also:

- Determine the life span of the affected lung cells

- Identify the "parent cells" that produce CFTR cells

- Find out how long treatment should last and how often it needs to be repeated

The first CF gene therapy experiments have involved lung cells because these cells are readily accessible and because lung damage is the most common, life-threatening problem in CF patients. However, scientists hope that the technologies being developed for lung cells will be adapted to treat other organs affected by cystic fibrosis.

\section{In case of Diabetic Neuropathy}

Gene therapy shows promise in treating diabetic polyneuropathy, a disorder that commonly affects diabetics who've had the disease for many years, a new study finds. Researchers in Boston found that intramuscular injections of vascular endothelial growth factor (VEGF) gene may help patients with diabetic polyneuropathy.

The study included 39 patients who received three sets of injections of VEGF gene in one leg and 11 patients who received a placeboLoss of sensation and pain in the legs and feet, weakness, and balance problems are among the symptoms associated with diabetic neuropathy. The loss of sensation means that ulcerations on the feet may go undetected, which can lead to amputation. "Most patients had fairly severe neuropathy, and the expectation for improvement was therefore not high," Dr. Allan Ropper, executive vice chair of the neurology department at Brigham and Women's Hospital in Boston, said in a hospital news release. The VEGF gene used in the study is active without packaging it in a virus, which is a major safety advantage, according to the researchers."The study shows that this form of gene transfer therapy can be performed relatively safely, but further investigation using a larger study group is needed before it can be introduced as a mainstream therapy," Ropper said.

\section{In case of Metastatic Melanoma}

Since the discovery of DNA and the subsequent recognition that genetic defects (either inherited or acquired) can be responsible for various disease states, the concept of gene therapy has been extremely appealing. However, despite intensive work in this field of medicine, gene therapy has yet to make a major impact on the treatment of patients. Many technical challenges exist that must be overcome before gene therapy can be put into widespread practice.

The gene delivery system (vector) encounters extracellular and intracellular barriers, must be nontoxic and non immunogenic, and must allow sufficient expression of the gene of interest. Many vectors have been created in attempts to overcome these problems; however, the ideal expression vector for use in humans has yet to be identified. Both inherited and acquired diseases may potentially benefit from gene therapy. X-linked severe combined immunodeficiency- $\mathrm{X} 1$ is an inherited disease in which gene therapy is not only being actively pursued as a potentially curative treatment, but some exciting progress has been made in recent years.

\section{Future aspects of gene therapy}

- Nanotechnology + gene therapy yields treatment to torpedo cancer. March, 2009. The School of Pharmacy in London is testing a treatment in mice, which delivers genes wrapped in nanoparticles to cancer cells to target and destroy hard-toreach cancer cells. Read BBC article.

- Results of world's first gene therapy for inherited blindness show sight improvement. 28 April 2008. UK researchers from the UCL Institute of Ophthalmology and Moorfields Eye Hospital NIHR Biomedical Research Centre have announced results from the world's first clinical trial to test a revolutionary gene therapy treatment for a type of inherited blindness.

- Researchers at the National Cancer Institute $(\mathrm{NCl})$, part of the National Institutes of Health, successfully reengineer immune 
cells, called lymphocytes, to target and attack cancer cells in patients with advanced metastatic melanoma. This is the first time that gene therapy is used to successfully treat cancer in humans. See New Method of Gene Therapy Alters Immune Cells for Treatment of Advanced Melanoma (August 30, 2006).

- Gene therapy is effectively used to treat two adult patients for a disease affecting non lymphocytic white blood cells called myeloid cells. Myeloid disorders are common and include a variety of bone marrow failure syndromes, such as acute myeloid leukemia. The study is the first to show that gene therapy can cure diseases of the myeloid system. See Gene Therapy Appears to Cure Myeloid Blood Diseases In Groundbreaking International Study (March 31, 2006)..

- University of California, Los Angeles, research team gets genes into the brain using liposomes coated in a polymer call polyethylene glycol (PEG). The transfer of genes into the brain is a significant achievement because viral vectors are too big to get across the "blood-brain barrier." This method has potential for treating Parkinson's disease. See Undercover Genes Slip into the Brain (March 20, 2003).

- RNA interference or gene silencing may be a new way to treat Huntington's. Short pieces of double-stranded RNA (short, interfering RNAs or si RNAs) are used by cells to degrade RNA of a particular sequence. If a si RNA is designed to match the RNA copied from a faulty gene, then the abnormal protein product of that gene will not be produced. See Gene Therapy May Switch off Huntington's (March 13, 2003).

- New gene therapy approach repairs errors in messenger RNA derived from defective genes. Technique has potential to treat the blood disorder thalassaemia, cystic fibrosis, and some cancers. See Subtle Gene Therapy Tackles Blood Disorder (October 11, 2002).

- Researchers at Case Western Reserve University and Copernicus Therapeutics are able to create tiny liposomes 25 nanometers across that can carry therapeutic DNA [13]through pores in the nuclear membrane.Sickle cell is successfully treated in mice

\section{Conclusion}

- According to gene therapy different types of genetic disorder are cured. In case of cystic fibrosis, Diabetes, AIDS, Hepatitis melanoma, Alizhmer, Parkinson's diseses etc.

- In case of Parkinson's disease one trial is done Neurologix a biotech company announced that they have successfully completed its landmark Phase I trial of gene therapy[14] for Parkinson's Disease.

- 12 patient study with four patients in each of three dose escalating cohorts. All procedures were performed under local anesthesia and all 12 patients were discharged from the hospital within 48 hours of the procedure, and followed for 12 months. Primary outcomes of the study design, safety and tolerability, were successfully met. There were no adverse events reported relating to the treatment.[15]

Steps involved in treatment of Parkinson's disease

- In cas of Diabetes gene therapy also play a good role. Replacing a mutated gene that causes disease with a healthy copy of the gene.

- Inactivating, or "knocking out," a mutated gene that is functioning improperly.

- Introducing a new gene into the body to help fight a disease.

\section{References}

[1] Perez E.E., Wang J., Miller J.C. (2008) Nat. Biotechnol. 80816.

[2] Urnov F.D., Rebar E.J., Holmes M.C., Zhang H.S., Gregory P.D. (2010) Genome editing with engineered zinc finger nucleases. 636-646.

[3] Durai S., Mani M., Kandavelou K., Wu J., Porteus M.H., Chandrasegaran S. (2009) Nucleic Acids Res. 33 ,5978-90.

[4] Harwood, Adrian J. Protocols for Gene Analysis.

[5] Friedmann T., Roblin R. (1972) Science 175 (25), 949.

[6] Alvarez-Erviti L, Seow Y, Yin HF et al. (2011) Delivery of siR$N A$ to the mouse brain by systemic injection of targeted exosomes.

[7] Wrobel I. and Collins D. (1995) Biochim. Biophys. Acta, 1235, 296-304.

[8] Woods N.B., Bottero V., Schmidt M., von Kalle C., Verma I.M. (2006) Gene therapy: therapeutic gene causing lymphoma. Nature 440, 7088.

[9] Wang Hongjie, Dmitry M. Shayakhmetov, Tobias Leege, Michael Harkey, Qiliang Li, Thalia Papayannopoulou, George Stamatoyannopolous, and André Lieber (2005) Journal of Virology 79(17), 10999-101.

[10]Gao X. and Huang L. (1996) Biochemistry 35, 1027.

[11]Horn P.A., Morris J.C., Neff T., Kiem H.P. (2004) Mol. Ther. 10(3), 417-31.

[12]Jenkins R.G., Meng Q.H., Hodges R.J., Lee L.K., Bottoms S.E.W., Laurent G.J., Willis D., Ayazi Shamlou P., McAnulty R.J. and Hart S.L. Farhood H., Serbina N. and Huang L. (1995) Biochim. Biophys. Acta, 1235, 289-295.

[13]Chen Q.R., Zhang L., Stass S.A. and Mixson A.J. (2000) Gene Ther.

[14]Duguid J.G., Li C., Shi M., Logan M.J., Alila H., Rolland A., Tomlinson E., Sparrow J.T. and Smith L.C. (1998) Biophys. J. 74, 2802-2814.

[15]Brown M.D., Sch"atzlein A.G. and Uchegbu I.F. (2001) Int. J. Pharm. 229, 1-21.

[16]Tachibana R., Harashima H., Shinohara Y. and Kiwada H. (2001) Adv. Drug Delivery Rev. 52, 219-226.

[17]Auton C.W. (1998) Adv. Drug. Delivery Rev. 34, 51-64. 